\title{
$O$ risco de informação assimétrica sobre a liquidez dos contratos futuros de commodities agrícolas
}

\author{
The risk of asymmetric information on the liquidity of agricultural \\ commodities futures contracts
}

\author{
João Eduardo Ribeiro ${ }^{\dagger}$ \\ Antônio Artur de Souza \\ Eduardo de Abreu Moraes*
}

\begin{abstract}
Resumo O objetivo deste estudo é analisar o efeito do risco de assimetria informacional na liquidez dos contratos futuros de commodities agrícolas negociados pela B3. Para tanto, escolheu-se como amostra as negociações intradiárias das commodities Boi Gordo, Café Arábica, Milho e Soja, no período de 01 de dezembro de 2018 a 30 de novembro de 2019. A analise foi realizada por meio de um modelo de dados em painel, utilizando-se como proxy para liquidez, o spread, para a probabilidade de presença de assimetria informacional nas negociações a VPIN e, como variáveis de controle, o número de negócios, o volume negociado e a volatilidade. Pelo modelo estimado, demostrou-se que as variáveis consideradas são capazes de explicar $43,08 \%$ da variação do spread. Além disso, os resultados mostraram uma relação positiva entre o risco de se negociar com assimetria informacional e a liquidez de mercado, divergindo dos resultados encontrados na literatura nacional e internacional.
\end{abstract}

Palavras-chave: Assimetria informacional; Liquidez de mercado; Commodities agrícolas

Código JEL: G11, G12, G14.

\begin{abstract}
The aim of this study is to analyze the effect of the risk of informational asymmetry on the liquidity of agricultural commodity futures contracts traded on B3. To this end, we examine intraday negotiations of the commodities Live Cattle, Arabica Coffee, Corn, and Soybeans from December 1, 2018 to November 30, 2019. We carry out the analysis using a panel regression model. We use spread as a proxy for liquidity, VPIN as a proxy for the probability of informational asymmetry and, as control variables, the number of trades, volume traded, and volatility. Our results show that the regressors explain $43.08 \%$ of the spread variation. In addition, there is a positive relationship between the risk of trading with informational asymmetry and market liquidity, in contrast with the extant results in the literature.
\end{abstract}

Keywords: informational asymmetry; market liquidity; agricultural commodities JEL Code: G11, G12, G14.

Submitted on January 21, 2020. Revised on May 24, 2020. Accepted on June 2nd, 2020. Published online in July 2020. Editor in charge: Cristina Scherrer.

$\dagger$ Universidade Federal de Minas Gerais, Brazil: joaoribeiro.cco@gmail.com

$\ddagger$ Universidade Federal de Minas Gerais, Brazil: artur@face.ufmg.br

*Universidade Federal de Minas Gerais, Brazil: eduardoam@gmail.com 


\section{Introdução}

A liquidez de mercado é um fator que impacta no custo de transação dos ativos e no retorno exigido pelos investidores. Por causa disso, tem sido objeto de pesquisas desde meados da década de 1980 e, sua importância se dá no fortalecimento dos mercados e na redução dos custos de emissão e de transação. Nesse contexto, é fundamental estimar o efeito de diversos fatores sobre a liquidez, como por exemplo, a eficiência de mercado, o sistema de negociação e de liquidação no mercado secundário, a transparência do mercado, a ampliação e a diversificação da base de investidores, e a assimetria informacional presente nas negociações.

Dentre esses fatores, a assimetria informacional ocorre quando há diferença entre as informações que os agentes detêm, ou seja, quando há um desbalanceamento de informação a respeito de uma transação entre os participantes (alguns têm mais informações que outros). Para Grossman e Stiglitz (1980), o grau de informação dos agentes é refletido no preço dos ativos e, por causa disso, os autores os classificaram como traders informados, aqueles que detêm informação relevante sobre o ativo negociado, e traders nãoinformados. Assim, o trader informado dispõe de uma vantagem em comparação ao não-informado.

Tanto a assimetria informacional quanto a liquidez de mercado são fatores estudados à luz da teoria de microestrutura de mercado, que em função do aumento das transações em alta frequência (High Frequency Trading - HFT), teve uma expansão significativa de estudos usando-a como pilar (O'Hara, 1995). No Brasil, os estudos de microestrutura de mercado têm buscado entender a relação liquidez-retorno no mercado acionário brasileiro (Correia, Amaral e Bressan, 2008); os efeitos que a contratação de market makers causam na liquidez do mercado acionário (Carvalho, Ribeiro e Correia, 2018; Perlin, 2013); o impacto da assimetria informacional no retorno requerido de ações no mercado acionário brasileiro (Siqueira, Amaral e Correia, 2017); bem como o efeito da assimetria informacional no mercado futuro (Barbosa, 2014), que segundo () apresenta uma série de particularidades, com valor ajustado diariamente, gerando mais liquidez ao mercado.

No mercado futuro, vendedores e compradores fixam o preço de determinado ativo para liquidação em uma data posterior. O comprador busca se proteger do risco de alta no preço do ativo, enquanto o vendedor busca se proteger do risco de queda (Hull, 2010). O objetivo é minimizar possíveis riscos de caixa ocasionados pelas variações no preço de um ativo (Ribeiro, Sousa e Rogers, 2006). Dentre os diversos ativos negociados nesse mercado, destacam-se os contratos de commodities agrícolas negociados pela Brasil 
Bolsa Balcão (B3), que despertam interesse tanto de investidores institucionais quanto de investidores pessoas físicas, seja pela possibilidade de especulação ou pela função de hedge contra possíveis oscilações. Por se tratar de produtos fundamentais, as commodities agrícolas têm uma grande relevância para a economia brasileira. Em 2017, por exemplo, segundo o Ministério da Agricultura, Pecuária e Abastecimento (MAPA) (2018), essas foram responsáveis por $44,1 \%$ das exportações nacionais. Dentre aquelas negociadas pela B3, quatro têm negociação diária durante todo o ano, com liquidação física e/ou financeira dos contratos, sendo essas, o Café Arábica, o Boi Gordo, o Milho e a Soja.

Além da importância dessas quatro commodities nas exportações e na balança comercial brasileira, desperta atenção o grande volume de contratos negociados. Somente nos seis primeiros meses de 2019 foram comercializados mais de 16 bilhões de reais em contratos futuros. Nos últimos cinco anos, o Boi Gordo, commodity mais negociada, ultrapassou o montante de 117 bilhões de reais em negociações. Já o Milho, segunda mais comercializada, superou o valor de 61 bilhões de reais. Juntas, estas quatro commodities movimentaram mais de 225 bilhões de reais em cinco anos (B3, 2019).

Demonstrada a relevância das commodities agrícolas para a economia brasileira e a importância de se entender a influência que a assimetria informacional exerce sobre a liquidez no mercado futuro brasileiro, especialmente para a tomada de decisão de empresas e de investidores, o objetivo deste trabalho consiste em analisar o efeito do risco de assimetria informacional na liquidez dos contratos futuros de commodities agrícolas negociados pela B3. Outros trabalhos, como os de Barbosa (2014) e Siqueira et al. (2017) já se propuseram a entender o efeito da assimetria de informação sobre a liquidez dos ativos, porém, esse estudo inova ao bucar entender esse efeito na liquidez dos contratos de commodities agrícolas, ativos tão importantes para a economia brasileira.

Para a melhor compreensão do conteúdo, além dessa introdução, esse trabalho conta ainda com outras quatro seções. A próxima apresenta o referencial teórico sobre microestrutura de mercado, assimetria informacional e liquidez. Na seção três são descritos os procedimentos metodológicos adotados. A seção quatro expõe a análise e interpretação dos resultados encontrados, e na última, são apresentadas as considerações sobre o trabalho, além de destacar suas limitações e sugestões para trabalhos futuros. 


\section{Referencial teórico}

\subsection{Microestrutura e liquidez de mercado}

A liquidez de mercado pode ser compreendida como o custo da imediata execução de uma ordem de compra ou venda. É considerada um fator essencial na análise e gestão de investimentos (Amihud \& Mendelson, 1986). O’Hara (1995) emprega definição similar, apontando os mercados líquidos como aqueles que proporcionam negociações com um mínimo de impacto sobre os preços. Apesar de ser um tema amplamente explorado por pesquisadores em finanças, ainda não há um consenso sobre as melhores proxies para o estudo da liquidez, e a relação que se estabelece entre a liquidez e o retorno dos ativos.

Demsetz (1968) sugere que a liquidez de mercado seja medida por meio da proxy spread. Chordia, Roll e Subrahmanyam (2001) utilizaram um conceito similar em seus estudos ao pesquisarem os spreads do mercado e as atividades de negociação nas ações de empresas americanas entre 1993 e 1998. Autores como Amihud e Mendelson (1986) utilizam o spread como medida da iliquidez do ativo e encontram uma relação negativa entre liquidez e retorno. Ding (1999) analisou os fatores determinantes do spread e constatou que o número de negócios e a volatilidade dos preços (medida de risco) são os principais fatores. Vieira eMilach (2008) utilizam outras medidas para a liquidez do ativo, como o volume, volatilidade e turnover e encontram uma relação positiva entre liquidez e retorno. A relação entre liquidez e retorno dos títulos está atrelada à hipótese de aversão ao risco dos investidores. Assim, a dificuldade e os custos para transacionar um título sem liquidez significam maiores riscos para o investidor, que demanda um retorno maior para tal ativo (Machado e Medeiros, 2011).

Sobre o papel da liquidez de mercado, Gonçalves e Sheng (2010, p. 2) destacam que "entre seus inúmeros benefícios, a liquidez fortalece os mercados, aumenta o número de participantes, cria curvas de referência e, principalmente, reduz custos de transação e de emissão". Kunkel et al. (2014) entendem a liquidez como um fator primordial do mercado financeiro devido a uma possível relação entre a liquidez e o custo de capital das empresas. No que concerne os custos de transação, Amihud e Mendelson (1991) afirmam que quanto mais tempo o investidor permanecer com o ativo em sua carteira, maior será a amortização dos custos de transação. Nesse sentido, ativos com menor liquidez são mais interessantes para investidores de longo prazo e vice-versa.

Devido à relevância da liquidez de mercado, diferentes autores têm se 
empenhado ao seu estudo direto, ou incorporam a liquidez como uma variável explicativa nos modelos propostos em seus trabalhos, como por exemplo Amihud e Mendelsom (1986, 1991), Chordia et al. (2001), Jun, Marathe e Shawky. (2003), Correia et al. (2008), Machado e Medeiros (2011), Perobelli, Famá, e Sacramento (2016), Ribeiro, Carvalho, Maciel, Mendonça e Brandão (2018), dentre outros. A despeito do grande número de estudos sobre o tema, não há ainda na literatura um consenso sobre os efeitos da liquidez no retorno dos ativos.

\subsection{Relacionamento entre liquidez de mercado e toxicidade dos fluxos de ordens}

Ressaltando o papel da liquidez de mercado, O'Hara (2003) argumenta que a área de asset pricing ignora o objeto de estudo da microestrutura de mercado, isto é, o fato de o preço dos ativos evoluir no mercado. Para essa autora, a liquidez deveria ser incorporada nos modelos de precificação, senão deixariam de ser eficientes. Ela ressalta ainda que tanto a liquidez quanto a definição de preços estão relacionados ao grau de informação do mercado e a assimetria informacional entre os agentes.

Easley et al. (2011), ao estudarem o Flash Crash (quebra trilionária ocorrido nas bolsas de valores norte-americanas) e tratarem da relação assimetria informacional-liquidez, argumentaram que o Flash Crash foi causado por uma crise de liquidez, provocada principalmente pelas características estruturais do mercado de HFT. Em decorrência das grandes quantidades de ordens de compra e venda desse mercado e da necessidade de maior poder computacional, Easley et al. (2011) desenvolveram a Volume-Synchronized Probability of Informed Trading (VPIN), um modelo de estimação da assimetria informacional presente nos fluxos de ordens. Para validar empiricamente esse modelo, os autores apuraram a VPIN nos contratos futuros de E-mini S\&P 500 de janeiro de 2008 a outubro de 2010, destacando os dias próximos do Flash Crash. Os resultados demostraram que a VPIN indicou problemas de liquidez algumas horas antes da queda do índice Dow Jones, atingindo valor máximo minutos antes da queda brusca do índice.

Analisando apenas o dia do Flash Crash, Easley et al. (2011) mostraram que a VPIN obteve valor máximo no momento da queda do índice, mantendose alta até o final do dia e diminuindo à medida que o índice voltava ao patamar anterior à queda. Os autores explicam que nos mercados de HFT as negociações são gerenciadas por algoritmos, que emitem ordens de compra e de venda e que, por causa disso, as grandes provedoras de liquidez nesses mercados, são as empresas de HFT. Dessa forma, ao perceberem que se en- 
contram diante de possíveis perdas relacionadas à assimetria informacional nas negociações, reduzem ou liquidam suas posições, gerando queda de liquidez, como ocorreu no Flash Crash. Abad e Yagüe (2012) reforçam a ideia de Easley et al. (2011) ao apontarem que a assimetria informacional enfatiza a perda esperada de um market maker ao estar em um mesmo ambiente de um trader informado, isto é, na probabilidade dos market makers serem alvos de seleção adversa.

Em outra pesquisa que procurou explicar a relação entre assimetria informacional e liquidez, Easley et al. (2012) utilizaram dados intradiários com intervalos de um minuto e calcularam a VPIN dos contratos futuros da E-mini S\&P 500 de $1^{\circ}$ de janeiro de 2008 a 15 de agosto de 2011 . Neste estudo foi introduzido o conceito de mercado tóxico ao se referirem ao momento em que os market makers fornecem liquidez ao mercado sem saberem que estão em desvantagem na operação, ou seja, negociam com traders informados e fornecem liquidez com prejuízo. Os autores defendem o procedimento de cálculo como vantagem de a VPIN ser utilizada como métrica de toxicidade, o seu procedimento de cálculo, que não depende de estimativas intermediárias de parâmetros não observáveis, além de ser atualizada com dados intradiários ajustados ao volume. No estudo em questão, ficou demostrado que a VPIN tem poder de previsão significativo sobre a volatilidade induzida pela toxicidade, o que a torna uma ferramenta de gestão de risco para os mercados de HFT.

Além de Easley et al. (2011, 2012), outros estudos também buscaram compreender a relação entre a assimetria informacional e a liquidez dos mercados. Em um desses estudos, Duarte e Young (2009) retomam Glosten e Milgrom (1985) ao averiguarem que o impacto da assimetria informacional em grandes economias pode ser diversificável em virtude da existência do grande número de ativos negociados, que reduz, a vantagem dos traders informados sobre os não informados. Não obstante, em economias emergentes há possivelmente, uma maior probabilidade de ganhos anormais por traders informados por meio da obtenção de informação privada, em consequência da concentração do capital das firmas entre poucos investidores.

De acordo com Agudelo et al. (2015), os modelos de microestrutura de mercado implicam que a negociação informada reduz a liquidez e move os preços na direção da informação. Siqueira et al. (2017) argumentam que o provimento de liquidez pelos market makers se dá de maneira complexa, uma vez que os traders tem informações privadas a respeito de um ativo, e os market makers não. Em mercados de HFT, os market makers buscam obter pequenos ganhos que se expandem em transações com grandes quantidades 
de ordens e tais ganhos dependem exclusivamente do controle sobre o risco de serem alvo de seleção adversa. A probabilidade de ganho desses market makers ao transacionarem grandes quantidades de ativos aumenta quando há equilíbrio entre os fluxos de ordens. Quando não existe esse equilíbrio, há chances de os market makers serem alvos de seleção adversa e, consequentemente, devido à alta toxicidade, liquidarem suas posições, diminuindo a liquidez do mercado.

Aslan, Easley, Hvidkjaer e O'Hara (2011) ao investigarem a relação entre contabilidade, microestrutura e precificação de ativos, mostram que a assimetria informacional afeta o retorno dos ativos. Para os autores, isso corrobora com a hipótese de que é a informação e não a liquidez que afeta o retorno.

Yoon, Zo e Ciganek (2011) investigaram se a adesão do Extensible Business Reporting Language (XBRL), uma estrutura de troca de informações comerciais, contribui para a diminuição do risco de assimetria informacional nas negociações. Os autores utilizaram dados de dezembro de 2007 a agosto de 2008 de 550 empresas listadas na Korea Exchange (KRX), e em suas análises, estimaram uma regressão múltipla na qual, além do spread, emprega o volume, o número de negócios e a volatilidade do preço das ações como variáveis de controle. Os resultados documentados pelos autores mostraram que existe uma associação significativa e negativa entre a adoção de XBRL e a assimetria informacional, isto é, a adoção do XBRL pode levar à redução da assimetria informacional no mercado acionário coreano. Os resultados ainda evidenciaram uma relação positiva entre o spread, o volume e a volatilidade, e uma associação negativa entre o spread e o número de negócios. Para Yoon et al. (2011), em um mercado eficiente, com baixa assimetria informacional, a volatilidade do preço dos ativos tende a ser baixa, uma vez que os problemas de seleção adversa entre os traders tende a diminuir.

Siqueira et al. (2017) analisaram por meio da VPIN, a relação entre a assimetria informacional e os retornos exigidos de portfólios no mercado acionário brasileiro. Os resultados mostraram um alto nível de toxicidade nos fluxos de ordens das ações. Com objetivo de testar o efeito do risco de informação assimétrica no retorno das ações, os autores incluíram a VPIN aos modelos de três e de cinco fatores de Fama e French $(1993,2015)$ e de quatro fatores de Carhart (1997). Os resultados apontaram que a VPIN funciona como um complemento ao fator tamanho e sua inclusão melhora o desempenho dos modelos, que apresentam um maior poder de explicação do risco informacional sobre o retorno dos portfólios.

Em um estudo mais recente, Foucault, Kozhan e Tham (2017) estudaram oportunidades de arbitragem de curta duração ocasionadas pelo atraso de 
ajuste de novas informações pelos terminais de negociação. Para tanto, os autores utilizaram dados do mercado cambial dólar-euro, dólar-libra e libra-euro da Reuters D-3000, uma das principais plataformas de negociação interdealer utilizadas pelos traders. Como resultados, esses autores mostraram que a iliquidez é maior nos dias em que a fração de oportunidades de arbitragem tóxica e a velocidade relativa dos arbitradores são maiores. A demora no processamento de informações, mesmo que de segundos, gera ganho de eficiência de preço da arbitragem de alta frequência e aumento do custo do risco de seleção adversa, evidenciando uma relação direta entre assimetria informacional e iliquidez.

\section{Metodologia}

A população objeto desse trabalho é composta pelos fluxos de ordens de compra e venda dos contratos futuros das commodities agrícolas negociadas pela B3. A amostra foi constituída pelas negociações intradiárias dos contratos futuros de Boi Gordo (BGI), Café Arábica (ICF), Milho (CCM) e Soja (SJC), no período de 01 de dezembro de 2018 a 30 de novembro de 2019. Foram selecionados todos os contratos negociados no período de analise, sem distinção de vencimento e, construído assim, uma série contínua de ordens. Tal procedimento foi adotado uma vez que, o vendimento dos contratos não influencia a hipótese de que a alta probabilidade de negociação informada de um mercado (altos níveis de VPIN) influencia o comportamento dos investidores, que liquidam suas posições e impactam dessa forma, a liquidez de mercado.

A escolha destes quatro tipos de contratos se deu pelo fato de terem negociações durante todo o ano, com possibilidade de liquidação financeira e/ou física, e o período foi determinado em função da disponibilização dos dados pelo portal Market Data da B3, no qual foram coletados. O período de 12 meses é comum em estudos envolvendo dados intradiários, uma vez que o número de ordens de compra e venda é muito relevante. O tratamento dos dados, bem como a obtenção das variáveis foram realizadas via linguagem de programação python. Já o modelo e os testes de diagnóstico foram estimados via software STATA.

Como proxy para a liquidez, foi utilizado o bid-ask-spread (Spread), definido por Demsetz (1968) como a diferença entre a menor oferta de venda (ask) e a maior oferta de compra (bid), logo antes da negociação (Spread $\tau=$ ask $\tau-b i d \tau)$. Ressalta-se que esta proxy adotada é uma medida de iliquidez, nesse sentido, quanto maior for o spread entre o bid e o ask de um título, menor a sua liquidez. O bid-ask-spread foi obtido via linguagem de progra- 
mação em python, utilizando os dados disponíveis nos arquivos do Market Data. Tais arquivos dispõem de uma coluna que identifica o lado agressor da ordem, em compra ou venda.

Todas as variáveis, inclusive o bid-ask-spread, foram obtidas por bucket de negociação, os mesmos utilizados na obtenção da VPIN, o que garantiu que o número de observações fosse igual para todas as variáveis. Cada bucket equivale a um cinqüenta avos do volume diário de negociação e, para se obter uma observação de bid-ask-spread por bucket, utilizou-se da menor oferta de venda (ask $\tau)$ menos a maior oferta de compra (bid $\tau)$ dentro de cada bucket. A escolha do bid-ask-spread como proxy para a liquidez se deu inspirada nos trabalhos que utilizaram essa mesma proxy, como Akay, Cyree, Griffiths e Winters (2012), Easley, Engle, O'Hara e Wu (2008), Easley et al. (2012), Siqueira et al. (2017), dentre outros.

Quanto à assimetria informacional, Easley et al. (2012) propõem a métrica VPIN para medir a toxicidade dos fluxos de ordens em um ambiente de negociação em alta frequência. A equação (1) representa o modelo para se obter a VPIN, proposto por Easley et al. (2012), em que: $V_{\tau}^{S}$ é o volume de ordens de venda de cada bucket; $V_{\tau}^{B}$ é o volume de ordens de compra de cada bucket; $V$ é o volume de cada bucket e $n$ é o número de buckets utilizados para aproximar o desequilíbrio esperado.

$$
\mathrm{VPIN}=\frac{\sum_{\tau=1}^{n}\left|V_{\tau}^{S}-V_{\tau}^{B}\right|}{n V}
$$

Nesse sentido, a estimação da VPIN carece da determinação das variáveis $V$ e $n$. Para se calcular a VPIN de um dia, têm-se $V$ como sendo um cinquenta avos do volume de negociação diária (bucket) e $n$ como sendo 50. Para se calcular a VPIN semanal por exemplo, a variável $n$ passaria a ser 250. De acordo com Easley et al. (2012), espera-se que a velocidade de atualização da VPIN simule a velocidade de informações ao mercado. Para a classificação das ordens em compra ou venda, Easley et al. (2012) optaram pela utilização do tick-rule (TR), um dos algoritmos de classificação mais utilizados na literatura. Neste trabalho, optou-se pela utilização da classificação real fornecida pela B3.

Para apurar uma possível relação de causa e efeito entre a assimetria informacional (VPIN) e a liquidez de mercado (bid-ask-spread) em negociações de commodities agrícolas do mercado futuro da B3, foi utilizado um modelo de dados em painel para toda a amostra. Procedimento adotado também por Agudelo et al. (2015) para explicar a relação entre assimetria informacional e liquidez nos seis maiores mercados de ações da América Latina. 
Esse tipo de modelo apresenta diversas vantagens em relação àquelas de corte transversal e de séries temporais, como por exemplo o fato de controlarem a heterogeneidade presente nos dados, permitirem o uso de mais observações, aumentarem o número de graus de liberdade, diminuírem a colinearidade entre as variáveis, além de serem capazes de mensurar e identificar efeitos que não são possíveis de serem detectados por meio da análise de dados em corte transversal ou de séries temporais (Duarte, Lamounier e Takamatsu, 2007).

Na estimação do modelo, além da utilização da VPIN como variável independente, foram utilizadas as variáveis volume (VOL), número de negócios (NEG) e volatilidade do preço (VLA) como variáveis de controle (Ding, 1999; Yoon, et al. 2011). Estas variáveis também foram obtidas por bucket de negociação, os mesmos utilizados na obtenção da VPIN. O VOL e o NEG são fornecidos no arquivo do Market Data da B3, já a VLA foi calculada pela diferença entre o preço mais alto e o preço mais baixo, dividido pela média dos preços, procedimento também empregado por Yoon et al. (2011). O modelo geral estimado é representado pela equação (2).

$$
|\mathrm{Spread}|_{i t}=\beta_{0 i t}+\beta_{1 i t} \mathrm{VPIN}_{i t}+\beta_{2 i t} \mathrm{NEG}_{i t}+\beta_{3 i t} \mathrm{VOL}_{i t}+\beta_{4 i t} \mathrm{VLA}_{i t}+e_{i t}
$$

em que $\beta_{0}$ refere-se ao parâmetro de intercepto; $\beta_{1}, \beta_{2}, \beta_{3}$ e $\beta_{4}$ referemse aos coeficientes correspondentes a cada uma das variáveis explicativas do modelo; $e_{\text {it }}$ refere-se ao erro idiossincrático, uma vez que varia aleatoriamente para todos os indivíduos e períodos; $i$ representa o indivíduo, que nesse caso são os dados de negociação das comodities; e $t$ representa o período que está sendo analisado. $\mathrm{O}$ valor absoluto do spread foi utilizado devido à necessidade de se medir a distância do resultado à zero, e assim ter a dimensão da iliquidez.

Para Wooldridge (2010), há três modos de estimação para modelos de dados em painel: (i) modelo de efeitos fixos, em que admite-se haver no conjunto de dados características distintas e constantes no tempo; (ii) modelo de efeitos aleatórios, que trata os efeitos específico-individuais como variáveis aleatórias, supondo não haver correlação entre os efeitos individuais e as demais variáveis aleatórias; e (iii) modelo pooled data (dados empilhados), a técnica mais simples e ingênua por desconsiderar as dimensões de tempo e espaço.

Por causa das opções, Wooldridge (2010), aponta ser importante realizar testes estatísticos para definir qual modelo melhor se adapta a amostra do estudo. Nesse sentido, foi utilizado o teste de Hausman para a confrontação entre o modelo de efeitos fixos e efeitos aleatórios, o teste de Chow para a comparação entre o modelo pooled data e o modelo de efeitos fixos e o teste 
Tabela 1

Estatísticas descritivas do bid-ask-spread

\begin{tabular}{lrrrr}
\hline & BGI & ICF & CCM & SJC \\
\hline mínimo & $-56,000$ & $-25,600$ & $-10,850$ & $-0,590$ \\
máximo & 24,250 & 27,250 & 7,220 & 0,790 \\
média & $-0,057$ & 0,053 & $-0,040$ & 0,000 \\
desvio padrão & 1,849 & 2,145 & 0,792 & 0,043 \\
\hline
\end{tabular}

de Breusch-Pagan para a comparação entre o modelo de efeito aleatório e o modelo pooled data. Além dos testes para a escolha do modelo, foi verificado, através dos testes de normalidade dos resíduos, homoscedasticidade, autocorrelação e multicolinearidade, se o modelo cumpre os pressupostos exigidos.

\section{Análise e discussão dos resultados}

Em busca de cumprir com o objetivo de analisar o efeito do risco de assimetria informacional na liquidez dos contratos futuros das commodities agrícolas, foram coletados dados intradiários das negociações das commodities BGI, ICF, CCM e SJC, que tiveram transações diárias durante o período 01/12/2018 a 30/11/2019. A partir dos dados disponíveis foi possível calcular as variáveis e viabilizar as análises.

\subsection{Estatísticas descritivas}

A Tabela 1 traz as estatíscitas descriticas para o spread para cada commoditie analisada. Os contratos de SJC foram os mais líquidos, apresentando média de spread de 0 e desvio padrão de 0,043 . Os maiores valores foram observados nos contrados de BGI, com valor mínimo de -56 e máximo de 24,25. As commodities ICF e CCM tiveram média de 0,053 e-0,040, respectivamente.

O resultado demostra uma alta liquidez no mercado futuro de commodities agrícolas, e vai ao encontro de Amihud e Mendelson (1986), que encontraram um bid-ask-spread médio de 0,025 no mercado americano, resultado próximo aos encontrados para as commodities analisadas. Perobelli et al. (2016) ao estudarem bid-ask-spread médio de -0,329 e Ribeiro, Souza, Carvalho e Amaral (2019), ao analisarem as negociações das Brazilian Depositary Receipts (BDRs) entre 2010 e 2017, encontraram um valor médio de 3,03, resultados superiores aos encontrados neste trabalho.

No que concerne à VPIN, a Tabela 2 traz as estatísticas descritivas e mos- 
Tabela 2

Estatísticas descritivas da VPIN

\begin{tabular}{lrrrr}
\hline & BGI & ICF & CCM & SJC \\
\hline mínimo & 0,000 & 0,000 & 0,000 & 0,000 \\
máximo & 1,000 & 1,000 & 1,000 & 1,000 \\
média & 0,774 & 0,670 & 0,555 & 0,951 \\
desvio padrão & 0,319 & 0,353 & 0,324 & 0,182 \\
\hline
\end{tabular}

tra que o maior valor médio encontrado foi de 0,951 para a commodity SJC e o menor foi de 0,555 para a a commodity CCM. O resultado mostra uma maior probabilidade de negociação informada nos contratos futuros de SJC. Para as commodities ICF e BGI, os valores médios de VPIN foram de 0,67 e 0,774, respectivamente. Em todas as commodities, conforme esperado e devido à métrica de Easley et al. (2012), os valores mínimos e máximos foram de 0 e 1 respectivamente.

Dos trabalhos que utilizaram a VPIN para mensurar a assimetria informacional nos fluxos de ordens, Abad e Yagüe (2012) utilizaram uma amostra de 15 ações comercializadas no mercado espanhol durante o ano de 2009 e as dividiram pelo valor de mercado em pequenas, médias e grandes ações. Os autores obtiveram VPIN média de 0,532; 0,402 e 0,254 para os grupos pequeno, médio e grande, respectivamente. Borochin e Rush (2016), ao estudarem o mercado norte-americano por uma amostra com ações avaliadas acima de cinco dólares no período de 1993 a 2013, encontraram VPIN média de 0,1774 . No mercado acionário brasileiro, Siqueira et al. (2017) constataram uma VPIN média de 0,4548 , encontrando-se o maior número de buckets entre 0,2 a 0,4 . Nos três estudos, portanto, a VPIN encontrada foi abaixo dos valores desse trabalho, sugerindo que o mercado futuro brasileiro de commodities agrícolas apresentou maior probabilidade de negócios informados do que estes mercados.

No tocante ao mercado futuro, Easley et al. (2011, 2012) estudaram o Flash Crash e calcularam a VPIN para o índice E-mini S\&P 500. No trabalho de 2011, esses autores analisaram o período de 01 de janeiro de 2008 a 30 de outubro de 2010, e encontraram a VPIN abaixo de $0,44 \mathrm{em} 80 \%$ dos buckets. Já Easley et al. (2012), utilizando dados de 01 de janeiro de 2008 a 15 de agosto de 2011, obtiveram VPIN média de 0,2251. Isso demostra que mesmo para o mercado futuro, nesses dois exemplos específicos, nos Estados Unidos, a probabilidade de negócios informados foi inferior à encontrada nesse trabalho. Esse resultado corrobora com Duarte e Young (2009), por afirmarem que o impacto da assimetria informacional é maior em economias 
Tabela 3

Matriz de correlação das variáveis

\begin{tabular}{lrrrr}
\hline variáveis & VPIN & NEG & VOL & VLA \\
\hline VPIN & 1,000 & & & \\
NEG & $-0,292$ & 1,000 & & \\
VOL & $-0,146$ & 0,688 & 1,000 & \\
VLA & $-0,078$ & 0,039 & 0,111 & 1,000 \\
\hline
\end{tabular}

Tabela 4

Fiv das variáveis independentes

\begin{tabular}{lcccc}
\hline variáveis & VPIN & NEG & VOL & VLA \\
\hline FIV & 1,107 & 2,054 & 1,938 & 1,021 \\
1/FIV & 0,903 & 0,487 & 0,516 & 0,980 \\
\hline
\end{tabular}

emergentes, uma vez que é maior a probabilidade de ganhos anormais por traders informados, como consequência da concentração do capital entre poucos investidores.

\subsection{Validação dos modelos}

Essa subseção é dedicada à apresentação dos testes de validação do modelo estimado na subseção posterior. Na Tabela 3 é apresentado a matriz de correlação das variáveis independentes. A maior correlação entre essas variáveis acontece entre o VOL e o NEG, com um valor de 0,688. É possível observar ainda que, todas as variáveis de controle (NEG, VOL e VLA), apresentaram correlação negativa com a VPIN.

Além disso, são apresentados, na Tabela 4, os valores do fator de inflação da variância (FIV) para as variáveis estudadas. Conforme aponta Wooldridge (2011), mesmo sendo uma escolha arbitrária, assume-se normalmente na literatura, que valores superiores a 10 para o FIV indicam problemas de colinearidade. Dessa forma, não há indícios de problemas com colinearidade entre as variáveis estudadas.

Posto isto e, seguindo o recomendado por Wooldridge (2010) foram estimados os modelos pooled data, efeitos fixos e efeitos aleatórios. Em seguida foram realizados os testes para identificar o modelo mais adequado. Os pvalores encontrados são apresentados na Tabela 5, com a rejeição da hipótese nula pelo teste de Chow, sendo uma evidência favorável à utilização do efeitos fixo em detrimento ao modelo pooled data. Pelo teste de Breusch-Pagan, 
Tabela 5

Testes modelos de dados em painel

\begin{tabular}{lr}
\hline teste & $p$-valor \\
\hline Chow & 0,0000 \\
Hausman & 0,0000 \\
Breusch-Pagan & 1,0000 \\
\hline
\end{tabular}

Tabela 6

Testes de diagnóstico

\begin{tabular}{lr}
\hline teste & $p$-valor \\
\hline Normalidade dos resíduos & 0,0000 \\
White & 0,0000 \\
Wooldridge & 0,0001 \\
\hline
\end{tabular}

que permite a comparação entre o efeito aleatório e o pooled data, não foi possível a rejeição da hipótese nula, o que mostra que os efeitos aleatórios não foram significativos. Na comparação entre os modelos de efeitos fixos e efeitos aleatórios, o teste de Hausman também rejeitou a hipótese nula, indicando a utilização dos efeitos fixos. Nesse sentido, para o seguimento desse trabalho, foi adotado o modelo de efeitos fixos.

Escolhido o modelo de efeitos fixos, resta verificar, por meio dos testes de diagnóstico, se o modelo cumpre os pressupostos exigidos para que os coeficientes estimados estejam corretos. A Tabela 6 apresenta os p-valores desses testes. Para testar a normalidade, o teste de assimetria e curtose foi empregado e rejeitou-se a hipótese nula da presença de normalidade dos resíduos. No entanto, Wooldridge (2010) ressalta que se o tamanho da amostra for suficientemente grande, pelo teorema do limite central, os resíduos tendem a uma distribuição normal. Com isso, a inferência a partir do modelo não é invalidada. Quanto à presença de heteroscedastidade no modelo, foi utilizado o teste de White, pelo qual rejeitou-se a hipótese nula de que a variância dos resíduos é constante, ou seja, atestou presença de heteroscedasticidade no modelo. Por fim, com o propósito de testar a existência de autocorrelação no modelo, foi empregado o teste de Wooldridge, que também rejeitou a hipótese nula de que não há autocorrelação de primeira ordem nos dados.

Devido à presença de heteroscedasticidade e autocorrelação no modelo, para o seguimento da análise, foi considerado o modelo com os erros-padrão corrigidos pelo procedimento de Driscoll e Kraay (1998). 
Tabela 7

Resultado da regressão

\begin{tabular}{lrrrr}
\hline $\mid$ spread $\mid$ & coef. & erro padrão & \multicolumn{1}{l}{$t$} & $\operatorname{Pr}>|t|$ \\
\hline constante & 0,77990 & 0,3514 & 22,19 & 0,0 \\
VPIN & $-0,75390$ & 0,0300 & $-25,09$ & 0,0 \\
NEG & $-0,00250$ & 0,0002 & $-10,02$ & 0,0 \\
VOL & 0,00004 & 0,0000 & 12,46 & 0,0 \\
VLA & 0,12390 & 0,0162 & 7,63 & 0,0 \\
\hline $\operatorname{Pr}>F=0,0$ & R-squared $=0,4308$ & $n$ obs. $=47.315$ \\
\hline
\end{tabular}

\subsection{Análise do modelo}

Corrigidos os problemas de heteroscedasticidade e autocorrelação, a Tabela 7 apresenta os coeficientes, com o respectivo nível de significância, o teste $\mathrm{F}$ para a significância do modelo e o coeficiente de determinação. Em uma análise da significância global do modelo, por meio do teste F, vemos que a hipótese nula é fortemente rejeitada, dado o p-valor de 0,0. Dessa forma, é possível afirmar que conjuntamente esses fatores são significativos como variáveis explicativas para o spread das negociações de contratos futuros das commodities agrícolas analisadas. Sobre o coeficiente de determinação, observa-se que as variáveis consideradas são capazes de explicar 43,08\% da variação do spread. Apesar do alto poder explicativo, esse resultado pode indicar que além das variáveis analisadas, há outros fatores que influenciam o spread no mercado futuro de commodities agrícolas. Este resultado no entanto, corrobora os achados de Ding (1999) e Yoon et al. (2011).

Observa-se que, para uma variação de uma unidade na VPIN, a variação no bid-ask-spread é de -0,7539. Todos os parâmetros desse modelo mostraram-se estatisticamente significativos ( $\mathrm{p}$-valor $=0,0$ ). Ressalta-se que esta proxy é uma medida de iliquidez, nesse sentido, o resultado vai de encontro a Easley et al. (2011, 2012), mostrando que o risco de se negociar com assimetria informacional nesse mercado está positivamente correlacionado com a liquidez. Diferentemente desse trabalho, Easley et al. (2011, 2012) mostraram que a VPIN tem poder de previsão significativo sobre a volatilidade induzida pela toxicidade, o que torna a VPIN uma ferramenta de gerenciamento de risco para os mercados de HFT. Em outro estudo sobre o tema, Agudelo et al. (2015) utilizaram modelos de dados em painel para explicar a relação entre assimetria informacional e liquidez nos seis maiores mercados de ações da América Latina. Segundo esses, os modelos de microestrutura de mercado implicam que a negociação informada reduz a liquidez e move os preços na direção da informação. Além disso, eles chegaram a 
resultados que sugerem que a assimetria informacional está relacionada aos retornos dos ativos, o que contribuiu para a discussão sobre compreensão da formação de preços nos mercados emergentes. As evidências desse trabalho não corroboram com os resultados de Agudelo et al. (2015).

Em um estudo mais recente, Foucault et al. (2017) estudaram oportunidades de arbitragem de curta duração, ocasionadas por atrasos de ajuste de novas informações pelos terminais de negociação, e mostraram que a demora no processamento de informações, mesmo que de segundos, gera ganho de eficiência de preço da arbitragem de alta frequência e aumento do custo do risco de seleção adversa. O resultado dos autores indica uma relação direta entre assimetria informacional e iliquidez, o que não se observou nesse trabalho.

Dessa forma, o presente estudo concluí que o risco de informação assimétrica, diferentemente do retratado na literatura sobre o tema, parece influenciar positivamente a liquidez no mercado futuro de commodities. Nesse sentido, tomando-se por base Easley et al. (2012), foi utilizado a correlação de Pearson, que mede o grau e a direção da correlação entre duas variáveis de escala métrica $\left(\rho\left(V P I N_{\text {it }}\right.\right.$, Spread $\left.\left._{\text {it }}\right)\right)$, no intuito de observar se a VPIN está fortemente correlacionada com o spread. O resultado mostrou uma correlação fraca de $-0,2$, porém com alto nível de confiança $($ Sig. $=0)$ entre as variáveis. Esse resultado evidencia uma correlação linear negativa entre a VPIN e o spread no mercado futuro de commodities agrícolas, no entanto, essa relação é abaixo do valor de 0,4 encontrado por Easley et al. (2012). Em termos de correlação, esse resultado sugere uma fraca dependência do spread sobre a VPIN.

\section{Considerações finais}

A finalidade desse trabalho foi analisar o efeito do risco de assimetria informacional na liquidez dos contratos futuros de commodities agrícolas. Para isso, escolheu-se como amostra às commodities com possibilidade de liquidação física e/ou financeira e com negociação durante todo o ano, sendo essas: BGI, ICF, CCM e SJC. Para fins de análise, foram identificadas na literatura as possibilidades de variáveis que representassem os fatores, e assim, ficou definido o spread, representando a liquidez de mercado, como variável dependente, a VPIN, representando a probabilidade de presença de assimetria informacional nas negociações, como variável independente e, o NEG, VOL e VLA, como variáveis de controle. Os dados intradiários foram coletados no portal Market Data da B3, no período de 01 de dezembro de 2018 a 30 de novembro de 2019. A linguagem de programação python foi empregada para 
tratamento dos dados e, o software STATA utilizado na estimação do modelo.

No que concerne o spread, os contratos de SJC foram os mais líquidos, com média de 0 e desvio padrão de 0,043 . Os maiores valores foram observados nos contrados de BGI, com valor mínimo de -56 e máximo de 24,25 . As commodities ICF e CCM tiveram média de 0,053 e-0,040, respectivamente. Tais resultados evidenciaram uma alta liquidez no mercado futuro de commodities agrícolas e corroboram com os estudos de Amihud e Mendelson (1986), Perobelli, et. al. (2016) e Ribeiro et al. (2019). No tocante à VPIN, o maior valor médio encontrado foi de 0,951 para a commodity SJC e o menor foi de 0,555 para a a commodity CCM. O resultado mostra uma maior probabilidade de negociação informada nos contratos futuros de SJC. Para as commodities ICF e BGI, os valores médios de VPIN foram de 0,67 e 0,774 , respectivamente. Os valores de VPIN encontrados são superiores aos documentados por Duarte e Young (2009) e Easley et al. (2011, 2012).

Pelo modelo estimado mediante dados em painel, demostrou-se que as variáveis consideradas são capazes de explicar $43,08 \%$ da variação do bidask-spread, resultado que corrobora os achados de Ding (1999) e Yoon et al. (2011). Quanto ao risco de assimetria informacional, observou-se que uma variação de uma unidade na VPIN provoca uma variação de $-0,7539$ no bid-ask-spread. O resultado vai de encontro a Easley et al. (2011, 2012), mostrando que o risco de se negociar com assimetria informacional nesse mercado está positivamente correlacionado com a liquidez. Utilizado-se a correlação de Pearson entre a VPIN e o bid-ask-spread, evidenciou-se uma correlação fraca de -0,2, valor, abaixo do encontrado por Easley et al. (2012) no mercado futuro americano.

É importante ressaltar que o mercado de commodities difere do mercado a vista e que não foram encontrados estudos similares deste mercado em específico. À relevância do presente trabalho decorre da ausência de estudos específicos no mercado de commodities agrícolas para o país, e pela necessidade de entendimento dos vários aspectos que podem afetar a liquidez de mercado. A contribuição dos resultados encontrados está no fato de identificar a relação assimetria informacional-liquidez, que precisa ser estudada mais profundamente para melhor ser compreendida. Aponta-se como limitação desse trabalho a amostra contendo apenas uma pequena parte das negociações do mercado futuro (commodities agrícolas) e sugere-se para as próximas pesquisas, analisar a relação assimetria informacional-liquidez em outras commodities, como por exemplo os contratos de Dólar futuro e de Depósitos Interfinanceiros (DI), que são as commodities mais negociadas pela B3 (B3, 2019). 


\section{Referências}

Abad, D., eYagüe, J. (2012). From PIN to VPIN: An introduction to order flow toxicity, Spanish Review of Financial Economics 10(2): 74-83.

Agudelo, D. A., Giraldo, S., e Villarraga, E. (2015). Does PIN measure information? Informed trading effects on returns and liquidity in six emerging markets, International Review of Economics \& Finance 39: 149-161.

Akay, O. O., Cyree, K. B., Griffiths, M. D., e Winters, D. B. (2012). What does PIN identify? Evidence from the T-bill market, Journal of Financial Markets 15(1): 29-46.

Amihud, Y., e Mendelson, H. (1986). Asset pricing and the bid-ask spread, Journal of Financial Economics 17(2): 223-249.

Amihud, Y., e Mendelson, H. (1991). Liquidity, asset prices and financial policy, Financial Analysts Journal 47(6): 56-66.

Andrade, R. D. G. O. (2015). Relevância das diferenças entre contratos futuros e a termo: O caso do trio. Dissertação de mestrado, Fundação Getúlio Vargas, São Paulo, SP.

Aslan, H., Easley, D., Hvidkjaer, S., e O'Hara, M. (2011). The characteristics of informed trading: Implications for asset pricing, Journal of Empirical Finance 18(5): 782-801.

Brasil Bolsa Balcão (2019). Resumo das Operações. Recuperado de http://www.bmfbovespa.com.br/pt_br/servicos/market-data/ consultas/mercado-de-derivativos/resumo-das-operacoes/ estatisticas/.

Barbosa, D. A. B. L. (2014). Toxicidade no Mercado Brasileiro. Dissertação de mestrado, Instituto Nacional de Matemática Pura e Aplicada, Rio de Janeiro, RJ.

Borochin, P., e Rush, S. (2016). Identifying and Pricing Adverse Selection Risk with VPIN. Available at SSRN 2599871.

Carhart, M. M. (1997). On persistence in mutual fund performance, Journal of Finance 52(1): 57-82.

Carvalho, G. A., Ribeiro, J. E., e Correia, L. F. (2018). Market Makers: O impacto da Introdução de Agentes de Liquidez no Mercado Acionário Brasileiro, In IX Congresso Nacional de Administração e ContabilidadeAdCont 2018. 
Chordia, T., Roll, R., e Subrahmanyam, A. (2001). Market liquidity and trading activity, Journal of Finance 56(2): 501-530.

Cooper, D. R., e Schindler, P. S. (2003). Métodos de Pesquisa em Administração. Porto Alegre: Bookman.

Copeland, T. E., e Galai, D. (1983). Information effects on the bid-ask spread, Journal of Finance 38(5): 1457-1469.

Correia, L. F., Amaral, H. F., e Bressan, A. A. (2008). O efeito da liquidez sobre a rentabilidade de mercado das ações negociadas no mercado acionário brasileiro, Revista de Administração e Contabilidade da Unisinos 5(2): 109-119.

Creswell, J. W. (2007). Projeto de Pesquisa: Métodos Qualitativo, Quantitativo e Misto. Porto Alegre: Artmed.

De Cesari, A., Espenlaub, S., e Khurshed, A. (2011). Stock repurchases and treasury share sales: Do they stabilize price and enhance liquidity?, Journal of Corporate Finance 17(5): 1558-1579.

Demsetz, H. (1968). The cost of transacting, Quarterly Journal of Economics 82(1): 33-53.

Ding, D. K. (1999). The determinants of bid-ask spreads in the foreign exchange futures market: A microstructure analysis, Journal of Futures Markets: Futures, Options, and Other Derivative Products 19(3): 307-324.

Driscoll, J. C.; e Kraay, A. C. (1998). Consistent covariance matrix estimation with spatially dependent panel data, Review of Economics and Statistics 80(4): 549-560.

Duarte, J., e Young, L. (2009). Why is PIN priced?, Journal of Financial Economics 91(2): 119-138.

Duarte, P. C., Lamounier, W. M., e Takamatsu, R. T. (2007). Modelos econométricos para dados em painel: Aspectos teóricos e exemplos de aplicação à pesquisa em contabilidade e finanças. In Congresso USP de Iniciação Científica em Contabilidade (Vol. 4, pp. 1-15).

Easley, D., Engle, R. F., O'Hara, M., e Wu, L. (2008). Time-varying arrival rates of informed and uninformed trades, Journal of Financial Econometrics 6(2): 171-207. 
Easley, D., De Prado, M. M. L., e O'Hara, M. (2011). The microstructure of the "flash crash": Flow toxicity, liquidity crashes, and the probability of informed trading, Journal of Portfolio Management 37(2): 118-128.

Easley, D., Prado, M. M. L., e O’Hara, M. (2012). Flow toxicity and liquidity in a high-frequency world, Review of Financial Studies 25(5): 1457-1493.

Fama, E. F. (1970). Efficient capital markets: A review of theory and empirical work, Journal of Finance 25(2): 383-417.

Fama, E. F., e French, K. R. (1993). Common risk factors in the returns on stocks and bonds, Journal of Economics 33(1): 3-56,

Fama, E. F., e French, K. R. (2015). A five-factor asset pricing model, Journal of Financial Economics 116(1): 1-22.

Foucault, T., Kozhan, R., e Tham, W. W. (2017). Toxic arbitrage, Review of Financial Studies 30(4): 1053-1094.

Glosten, L. R., e Milgrom, P. R. (1985). Bid, ask and transaction prices in a specialist market with heterogeneously informed traders, Journal of Financial Economics 14(1): 71-100.

Gonçalves, P. E., e Sheng, H. H. (2010). O apreçamento do spread de liquidez no mercado secundário de debêntures, Revista de Administração-RAUSP 45(1): 30-42.

Grossman, S. J., e Stiglitz, J. E. (1980). On the impossibility of informationally efficient markets, American Economic Review 70(3): 393-408.

Grullon, G., Kanatas, G., e Weston, J. P. (2004). Advertising, breadth of ownership, and liquidity, Review of Financial Studies 17(2): 439-461.

Hull, J. C. (2005). Fundamentos dos Mercados Futuros e de Opções: Manual de Soluções. São Paulo: Bolsa de Mercadorias \& Futuros.

Jun, S. G., Marathe, A., e Shawky, H. A. (2003). Liquidity and stock returns in emerging equity markets, Emerging Markets Review 4(1): 1-24.

Kunkel, F. I. R., Ceretta, P. S., Vieira, K. M., Girardi, V., e Righi, M. B. (2014). Comportamento temporal da liquidez no mercado brasileiro: Uma análise do período 1995-2012 através do modelo autoregressivo de mudanças Markovianas, Revista de Administração da UNIMEP 12(2): 21-41. 
Kyle, A. S. (1985). Continuous auctions and insider trading, Econometrica 53(6): 1315-1335.

Machado, M. A. V. (2011). Modelos de precificação de ativos e o efeito liquidez: Evidências empíricas do mercado acionário brasileiro, Revista Brasileira de Finanças 9(3): 383-412.

Madhavan, A. (2000). Market microstructure: A survey, Journal of Financial Markets 3(3): 205-258.

Ministério da Agricultura, Pecuária e Abastecimento. (2018). Superavit de US\$ 81,86 bilhões do agronegócio foi o segundo maior da história. Brasília. Recuperado de URL: http://www.agricultura.gov.br/noticias/superavit-de-us-81-86-bilhoesdo-agronegocio-foi-o-segundo-maior-da-historia.

O'Hara, M. (1995). Market Microstructure Theory. Cambridge: Blackwell Publishers.

O'Hara, M. (2003). Presidential address: Liquidity and price discovery, Journal of Finance 58(4): 1335-1354.

Perlin, M. (2013). Os efeitos da introdução de agentes de liquidez no mercado acionário brasileiro, Revista Brasileira de Finanças 11(2): 281-304.

Perobelli, F. F. C., Famá, R., e Sacramento, L. C. (2016). Return and liquidity relationships on market and accounting levels in Brazil, Revista Contabilidade \& Finanças 27(71): 259-272.

Ribeiro, J. E., Carvalho, G. A., Maciel, C. F., Mendonça, F. M., e Brandão, M. L. (2018). Produção científica brasileira sobre liquidez de mercado: Um estudo bibliométrico, Revista de Finanças e Contabilidade da Unimep 5(1): 95-110.

Ribeiro, J. E., Souza, A. A., Carvalho, G. A., e Amaral, H. F. (2019). The impact of the introduction of market makers on the negotiations of the Brazilian depositary receipts, Revista Catarinense da Ciência Contábil 18(54): 1-16.

Ribeiro, K. C. S., Sousa, A. F., e Rogers, P. (2006). Preços do café no Brasil: Variáveis preditivas no mercado à vista e futuro, REGE Revista de Gestão 13(1): 11-30.

Sampieri, R. H., Collado, C. F. e Lucio, P.B. (2006). Metodologia de Pesquisa. São Paulo: MacGraw-Hill. 
Siqueira, L. S., Amaral, H. F., e Correia, L. F. (2017). The effect of asymmetric information risk on returns of stocks traded on the BM\&FBOVESPA, Revista Contabilidade \& Finanças 28(75): 425-444.

Stoll, H. R. (1978). The supply of dealer services in securities markets, Journal of Finance 33(4): 1133-1151.

Vieira, K. M., e Milach, F. T. (2008). Liquidez/iliquidez no mercado brasileiro: Comportamento no período 1995-2005 e suas relações com o retorno, Revista de Administração e Contabilidade da Unisinos 5(1): 5-16.

Wooldridge, J. M. (2010). Introdução à Aconometria: Uma Abordagem Moderna. São Paulo: Cengage Learning.

Yoon, H., Zo, H., e Ciganek, A. P. (2011). Does XBRL adoption reduce information asymmetry?, Journal of Business Research 64(2): 157-163. 